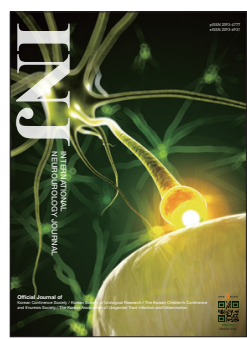

\title{
Neurourology Based on Original Perspectives and Novel Applications
}

\author{
Su Jin Kim (iD) https://orcid.org/0000-0002-1917-2780 \\ Department of Urology, Yonsei University Wonju College of Medicine, Wonju, Korea \\ Email: hygeisujin@naver.com
}

This issue of the International Neurourology Journal (INJ) deals with several important and interesting topics. With the increasing number of the prostate cancer patients, lower urinary tract symptoms (LUTS) are commonly observed in prostate cancer patients, in whom they constitute an important problem associated with low quality of life. Therefore, it is important to evaluate patients' LUTS. Moreover, most men with prostate cancer are likely to be affected by disorders such as benign prostate hyperplasia and overactive bladder (OAB), and these voiding problems can contribute to LUTS in prostate cancer patients. Walker et al. [1] reported preoperative urodynamic study (UDS) results in prostate cancer patients who underwent robotic and open surgery. They found that preoperative detrusor overactivity and other abnormalities during the filling phase were strongly associated with $\mathrm{OAB}$. However, the International Prostate Symptoms Score did not show strong correlations with UDS findings. In addition, the association of LUTS with radiation therapy for prostate cancer was reviewed [2].

A midurethral sling (MUS) is an effective treatment for stress urinary incontinence (SUI) in women, but some patients experience recurrence of SUI. Salvage therapies for MUS failure include transurethral bulking agent injections, tape shortening, repeated MUS, a pubovaginal sling using autologous fascia, and Burch colposuspension [3]. At present, there is no general consensus regarding which therapeutic method is more effective, and a therapeutic method should be selected according to the patient's characteristics. MUS is also an option for SU in men; therefore, problems associated with synthetic mesh can occur in men treated with MUS. Zhao et al. [4] investigated problems associated with synthetic MUS in male patients using the U.S. Food and Drug Administration Manufacturer and User Facility Device Experience database. In this study, they reported the 4 most common adverse, which were urinary incontinence, erosion, mechanical malfunction, and pain/numbness.

Pelvic pain is a chronic and complex conditions associated with interstitial cystitis/bladder pain syndrome (IC/BPS), pudendal neuralgia, gynecologic disorders (e.g., endometriosis and pelvic inflammatory disease), irritable bowel syndrome, and musculoskeletal problems. Pelvic pain is difficult to treat because it is associated with various etiologies and has complicated underlying mechanisms. Therefore, a patient-centered approach is necessary for the evaluation and treatment of pelvic pain. To improve researcher' and clinicians' understanding of neurourologic-associated pelvic pain, INJ presented perspectives about IC/BPS $[5,6]$

In addition to the above-mentioned research, this issue of INJ contains other novel and interesting studies. Li et al. [7] reported the effect of saphenous nerve stimulation combined with tibial nerve stimulation for the treatment of $\mathrm{OAB}$, and Shin et al. [8] observed improvement of near visual function after treatment with silodosin. Previous studies have reported that hypoxia and oxidative stress are associated with voiding problems and that some LUTS can be reduced by treatment for oxidative stress. The role of adenosine monophosphate-activated protein kinase in the regulation of hypoxia-induced apoptosis established in the study of Cho et al. [9] using bladder cancer cell may be a mechanism underlying for LUTS associated with oxidative stress. 
- Conflict of Interest: No potential conflict of interest relevant to this article was reported.

\section{REFERENCES}

1. Walker NF, Canagasingham A, Van Diepen D, Pirpiris A, Tse V, Leslie $S$, et al. Lower urinary tract functional assessment of men undergoing radical prostatectomy: correlation of preoperative clinical and urodynamic parameters. Int Neurourol J 2021;25:157-63

2. Kwon WA, Lee SY, Jeong TY, Moon HS. Lower urinary tract symptoms in prostate cancer patients treated with radiation therapy: past and present. Int Neurourol J 2021;25:119-27.

3. Kwon J, Kim Y, Kim DY. Second-line surgical management after midurethral sling failure. Int Neurourol J 2021;25:111-8.

4. Zhao H, Souders CP, Kuhlmann PK, Dallas K, Eilber K, Anger JT. Adverse events associated with synthetic male slings: an analysis of the food and drug administration manufacturer and user facility device experience database. Int Neurourol J 2021;25:172-6.
5. Ueda T, Hanno PM, Saito R, Meijlink JM, Yoshimura N. Current understanding and future perspectives of interstitial cystitis/bladder pain syndrome. Int Neurourol J 2021;25:99-110.

6. Lee YJu, Kim MM, Song SH, Lee S. A novel mobile acoustic uroflowmetry: comparison with contemporary uroflowmetry. Int Neurourol J 2021;25:150-6.

7. Li X, Wan X, Wang Z, Liang Y, Jia Z, Zhang X. Frequency-dependent effects on bladder reflex by saphenous nerve stimulation and a possible action mechanism of tibial nerve stimulation in cats. Int Neurourol J 2021;25:128-36.

8. Shin HJ, Choi WS, Lee SH, Lee AG, Kim A, Park HK, et al. Improvement in near vision following silodosin treatment in patients with lower urinary tract symptoms. Int Neurourol J 2021;25:164-71.

9. Cho TJ, Lee DH, Choi BH, Shinn HK, Park CS. Hypoxia-induced suppression of antiapoptotic Bcl-2 expression in human bladder tumor cells is regulated by caveolin-1-dependent adenosine monophosphate-activated protein kinase activity. Int Neurourol J 2021; 25:137-49. 